\title{
Seasonal Effects on a Beddington-DeAngelis Type Predator-Prey System with Impulsive Perturbations
}

\author{
Hunki Baek and Younghae Do \\ Department of Mathematics, Kyungpook National University, Daegu 702-701, South Korea \\ Correspondence should be addressed to Hunki Baek, hkbaek@knu.ac.kr
}

Received 3 March 2009; Accepted 7 August 2009

Recommended by Stephen Clark

\begin{abstract}
We study a Beddington-DeAngelis type predator-prey system with impulsive perturbation and seasonal effects. First, we numerically observe the influence of seasonal effects on the system without impulsive perturbations. Next, we find the conditions for the local and global stabilities of prey-free periodic solutions by using Floquet theory for the impulsive equation and small amplitude perturbation skills, and for the permanence of the system via comparison theorem. Finally, we show that seasonal effects and impulsive perturbation can give birth to various kinds of dynamical behavior of the system including chaotic phenomena by numerical simulations.
\end{abstract}

Copyright (C) 2009 H. Baek and Y. Do. This is an open access article distributed under the Creative Commons Attribution License, which permits unrestricted use, distribution, and reproduction in any medium, provided the original work is properly cited.

\section{Introduction and Model Formulation}

In ecology, one of main goals is to understand the dynamical relationship between predator and prey. Such relationship can be represented by the functional response which refers to the change in the density of prey attached per unit time per predator as the prey density changes. One of well-known functional responses is Beddington-DeAngelis functional response introduced by Beddington [1] and DeAngelis et al. [2], independently. It is similar to Holling type II functional response but contains an extra term describing mutual interference by predators. In fact, there are much significant evidences to suggest that functional responses with predator interference occur quite frequently in laboratory and natural systems [3]. Thus, we can establish a predator-prey model with Beddington-DeAngelis functional response as the following form $[1,4,5]$ :

$$
\begin{gathered}
x^{\prime}(t)=r x(t)\left(1-\frac{x(t)}{K}\right)-\frac{a x(t) y(t)}{b y(t)+x(t)+c^{\prime}}, \\
y^{\prime}(t)=-d y(t)+\frac{e a x(t) y(t)}{b y(t)+x(t)+c},
\end{gathered}
$$


where $x(t), y(t)$ represent the population densities of prey and predator at time $t$, respectively. In this system, the prey grows according to a logistic growth with intrinsic growth rate $r$ and $K$ is called the carrying capacity of the prey. For parameters setting, $a$ is the per-capita rates of predation of the predator, the constants $e, d$ are the conversion rate and the death rate of the predator, respectively, and the term by measures the mutual interference between predators.

It is necessary and important to consider models with periodic ecological parameters which might be quite naturally exposed such as those due to seasonal effects of weather or food supply [6]. Thus when the environmental factors that affect various parameters of the ecological model fluctuate periodically, then the corresponding parameters should be taken as periodic functions of time [7]. There are a number of ways to apply periodic perturbation in ecological models. Especially, one of the most popular ways to describe periodic phenomena is to use the sine (or cosine) wave or sinusoid function which describes a wave-like function of time with peak deviation from center and angular frequency [8-15]. Thus we consider the intrinsic growth rate $r$ in system (1.1) as periodically varying function of time due to seasonal variation and adopt the sine wave as mentioned above to investigate the seasonality on the system. In fact the seasonality is superimposed as follows:

$$
r_{0}=r(1+\epsilon \sin (\omega t))
$$

where the parameter $\epsilon$ represents the degree of seasonality; $\lambda=r \epsilon$ is the magnitude of the perturbation in $r_{0}, \omega$ is the angular frequency of the fluctuation caused by seasonality. Since $r_{0}$ is assumed to be positive, we have $0 \leq \epsilon \leq 1$. With this idea of periodic forcing, we consider the following predator-prey system with periodic variation in the intrinsic growth rate of the prey:

$$
\begin{gathered}
x^{\prime}(t)=r x(t)\left(1-\frac{x(t)}{K}\right)-\frac{a x(t) y(t)}{b y(t)+x(t)+c}+\lambda x(t) \sin (\omega t), \\
y^{\prime}(t)=-d y(t)+\frac{e a x(t) y(t)}{b y(t)+x(t)+c},
\end{gathered}
$$

where $\lambda$ and $\omega$ represent the magnitude and frequency of the forcing term, respectively. Of course, a number of researchers [14, 16, 17] have studied that dynamical systems with simple dynamic behavior in the constant parameter case display very complex behavior including chaos when they are periodically perturbed. In this context, in Section 2 we illustrate numerical simulations for system (1.3) to show the existence of limit circles and various kinds of dynamical behaviors including chaos. For this reason, system (1.3) reflects more realistic situation than system (1.1).

There are still some other periodic perturbations such as fire, flood, and mating habits or harvesting seasons which are not suitable to be considered continually. Suppose that with the pest outbreak, for example, there are many ways to beat agricultural pests. One of important ways is biological control leading reduction in pest population from the actions of other living organisms, often called natural enemies or beneficial species. As we know, anther important method for pest control is chemical control. Pesticides can reduce farmer's financial losses by preventing crop losses to insects and other pests. Such control tactics should be used not continuously but impulsively. There are many literatures on systems dealing with impulsive controls [10, 11, 13-15, 18-21]. Thus, we consider the 
following predator-prey system with adding periodic constant impulsive immigration of the predator regarded as natural enemy of the prey (pest) to system (1.3) and spraying pesticides (harvesting) on all species at the same times

$$
\begin{gathered}
x^{\prime}(t)=r x(t)\left(1-\frac{x(t)}{K}\right)-\frac{a x(t) y(t)}{b y(t)+x(t)+c}+\lambda x(t) \sin (\omega t), \\
y^{\prime}(t)=-d y(t)+\frac{e a x(t) y(t)}{b y(t)+x(t)+c}, \quad t \neq n \tau, \\
x\left(t^{+}\right)=\left(1-p_{1}\right) x(t), \quad t=n \tau, \\
y\left(t^{+}\right)=\left(1-p_{2}\right) y(t)+q, \\
\left(x\left(0^{+}\right), y\left(0^{+}\right)\right)=\left(x_{0}, y_{0}\right),
\end{gathered}
$$

where $\tau$ is the period of the impulsive immigration or stock of the predator, $0 \leq p_{1}, p_{2}<1$ present the fraction of the prey and the predator which die due to the harvesting or pesticides, and so forth, and $q>0$ is the size of immigration or stock of the predator.

If we take $b=0$, system (1.4) can be expressed as the Holling-type II predator-prey system with impulsive perturbations and seasonal effects as follows:

$$
\begin{gathered}
x^{\prime}(t)=r x(t)\left(1-\frac{x(t)}{K}\right)-\frac{a x(t) y(t)}{x(t)+c}+\lambda x(t) \sin (\omega t), \quad t \neq n \tau, \\
y^{\prime}(t)=-d y(t)+\frac{e a x(t) y(t)}{x(t)+c}, \\
x\left(t^{+}\right)=\left(1-p_{1}\right) x(t), \quad t=n \tau, \\
y\left(t^{+}\right)=\left(1-p_{2}\right) y(t)+q, \\
\left(x\left(0^{+}\right), y\left(0^{+}\right)\right)=\left(x_{0}, y_{0}\right) .
\end{gathered}
$$

While if $c=0$, then system (1.4) can be expressed as the ratio-dependent predator-prey system with impulsive perturbations and seasonal effects as follows:

$$
\begin{gathered}
x^{\prime}(t)=r x(t)\left(1-\frac{x(t)}{K}\right)-\frac{a x(t) y(t)}{b y(t)+x(t)}+\lambda x(t) \sin (\omega t), \quad t \neq n \tau, \\
y^{\prime}(t)=-d y(t)+\frac{e a x(t) y(t)}{b y(t)+x(t)}, \\
x\left(t^{+}\right)=\left(1-p_{1}\right) x(t), \quad t=n \tau, \\
y\left(t^{+}\right)=\left(1-p_{2}\right) y(t)+q, \\
\left(x\left(0^{+}\right), y\left(0^{+}\right)\right)=\left(x_{0}, y_{0}\right) .
\end{gathered}
$$

We will investigate system (1.4) together with systems (1.5) and (1.6). Impulsive differential equations such as (1.4) are found in almost every domain of applied science and have 


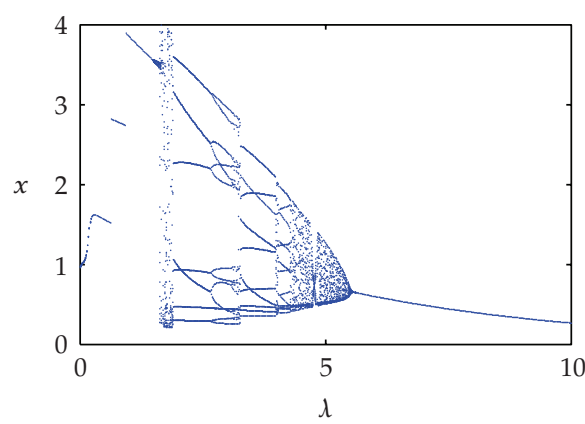

(a)

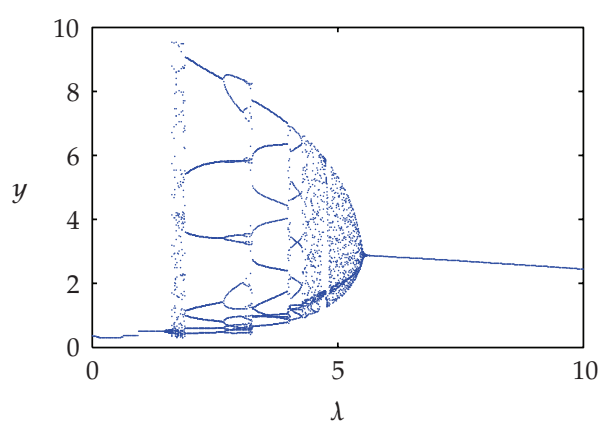

(b)

Figure 1: Bifurcation diagrams of system (1.3). (a) $x$ is plotted for $\lambda$, (b) $y$ is plotted for $\lambda$.

been studied in many investigations [10, 11, 14, 16, 17]. Especially, Zhang and Chen [15] considered system (1.4) when $p_{2}, \lambda=0$. They investigated abundance of complex dynamics for system (1.4) when $p_{2}, \lambda=0$ and suggested a more executable way for observing chaos and coexistence of attractors. They also gave a threshold that classifies between the permanence and the stability of prey-free solutions for system (1.4). However, in case $p_{2}, \lambda \neq 0$, system (1.4) has not been studied yet. Thus, the purpose of this paper is to find conditions for the stability of prey-free periodic solutions. Also, we show that the system is permanent under some conditions. In addition, using numerical simulations various kinds of dynamical phenomena are discussed in Section 4.

\section{Numerical Analysis of System (1.3)}

In this section we will numerically study the influence of the seasonality parameter $\lambda$ on system (1.3). For this, we fix parameters $\omega=2 \pi, a=3.2118, b=0.0246, c=3.3667, d=3.7798$, $e=3.3189, r=2.3553, K=9.7016$ and we choose $\left(x_{0}, y_{0}\right)=(1,1)$ as an initial point. It follows from [16] that system (1.3) with these parameters has a unique stable limit cycle when $\lambda=0$. Since the corresponding continuous system (1.3) cannot be solved explicitly and system (1.3) cannot be rewritten as equivalent difference equations, it is difficult to study them analytically. However, the influence of $\lambda$ may be documented by stroboscopically sampling one of the variables over a range of $\lambda$ values. Thus we numerically integrate system (1.3) and seek the behavior of the solutions. The bifurcation diagram provides a summary of essential dynamical behavior of system. Indeed the points that are plotted will represent either fixed or periodic sinks or other attracting sets including chaos. It shows the birth, evolution, and death of the attracting sets. In Figure 1, we illustrate bifurcation diagrams of system (1.3) to examine significant changes in the set of fixed or periodic points or other sets of interest. As is evident from Figures 1 and 2, the solutions are still periodic for values of $\lambda$ in the range $0 \leq \lambda<\lambda_{1}(\approx 1.5)$ and quasiperiodic motions appear when $\lambda>\lambda_{1}$ (see Figure 2(b)). Periodic windows are intermittently scattered. Also Figures $3(a)$ and $3(b)$ show the route to chaos through the cascade of period doubling. Moreover, although the magnitude $\lambda$ of seasonality increases, the solutions are stable and even they become periodic cycles like case $\lambda=0$ after $\lambda>\lambda_{2}(\approx 5.6)$ (see Figure 3(c)). We can also catch sight of the existence of occurrences of sudden changes in Figure 1 when $\lambda \approx 0.84,1.04,1.25,3.26,3.9$, and so forth. They can lead to nonunique attractors. For example, there exist at least three different attractors according 


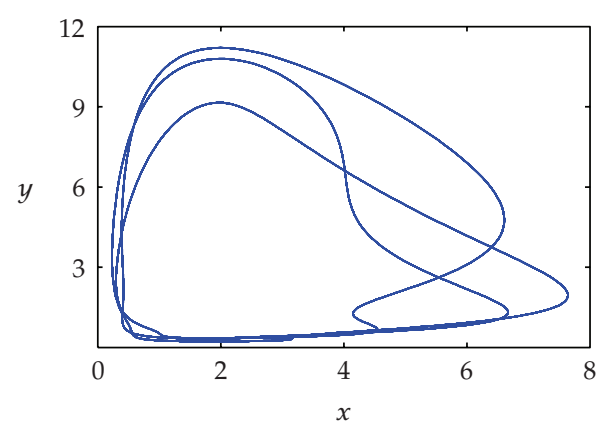

(a)

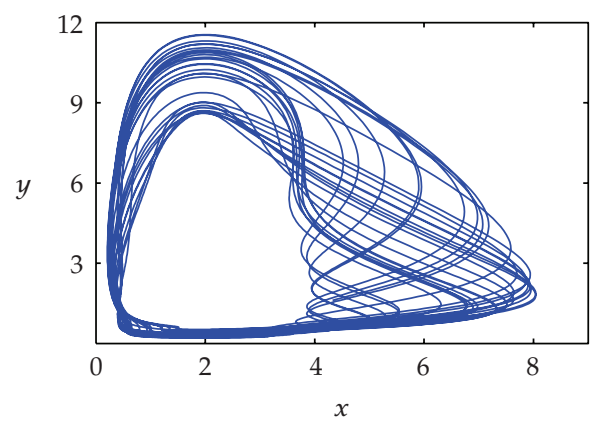

(b)

Figure 2: Phase portraits of solutions for system (1.3). (a) $\lambda=1.4$ (a $3 \tau$-periodic solution), (b) $\lambda=1.7$.

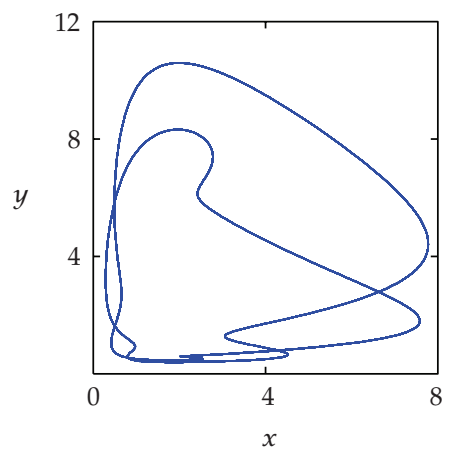

(a)

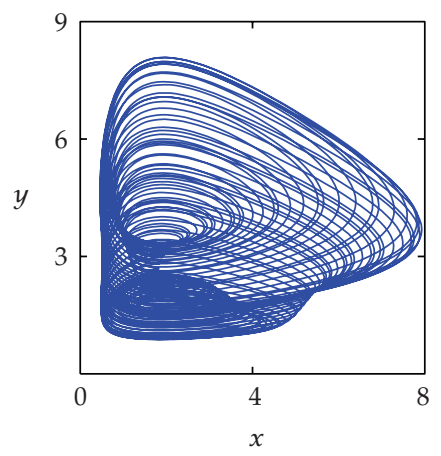

(b)

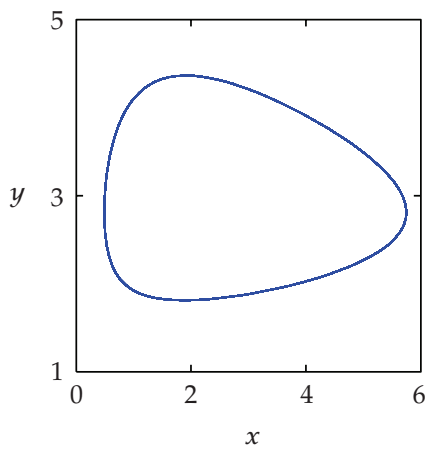

(c)

Figure 3: Phase portraits of solutions for system (1.3). (a) $\lambda=2.6$ (a $2 \tau$-periodic solution), (b) $\lambda=4.8$ (chaotic motion), (c) $\lambda=6.0$ (a $\tau$-periodic solution).

to initial values when $\lambda=3.9$ (see Figure 4 ). This result shows that the seasonality in just one parameter can give rise to multiple attractors. Thus, these numerical examples show that the dynamical behavior of system (1.3) is more abundant than that of system (1.1).

\section{Mathematical Analysis}

In this section we give some notations, definitions, and lemmas which will be useful for our main results.

Denote $\mathbb{N}$ the set of all of nonnegative integers, $\mathbb{R}_{+}=[0, \infty), \mathbb{R}_{+}^{*}=(0, \infty), \mathbb{R}_{+}^{2}=\{\mathbf{x}=$ $\left.(x, y) \in \mathbb{R}^{2}: x, y \geq 0\right\}$, and $f=\left(f_{1}, f_{2}\right)^{T}$ the right-hand side of system (1.4). Let $V: \mathbb{R}_{+} \times \mathbb{R}_{+}^{2} \rightarrow$ $\mathbb{R}_{+}$, then $V$ is said to be in a class $V_{0}$ if

(1) $V$ is continuous on $(n \tau,(n+1) \tau] \times \mathbb{R}_{+}^{2}$, and $\lim _{(t, \mathbf{y}) \rightarrow(n \tau, \mathbf{x}), t>n \tau} V(t, \mathbf{y})=V\left(n \tau^{+}, \mathbf{x}\right)$ exists;

(2) $V$ is locally Lipschitzian in $\mathbf{x}$. 


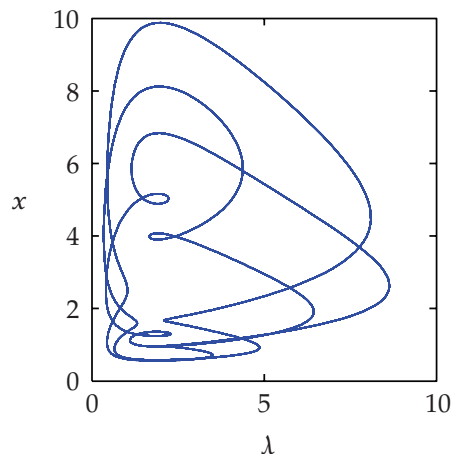

(a)

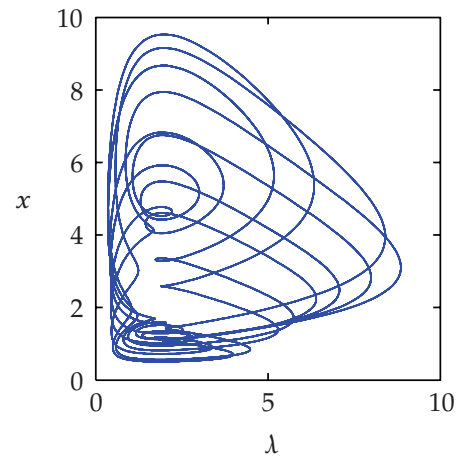

(b)

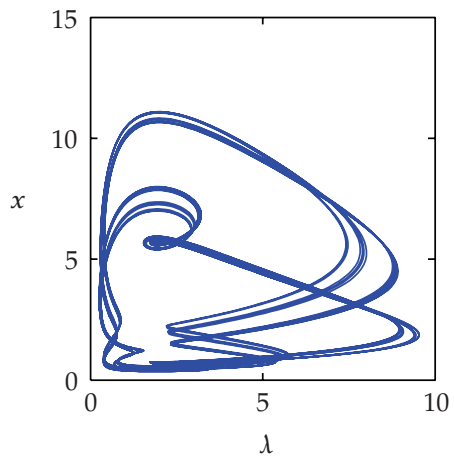

(c)

Figure 4: Coexistence of solutions when $\lambda=3.9$, and (a) $\left(x_{0}, y_{0}\right)=(1,1)$, (b) $(1.54,1.65)$, and (c) $(4.79,1.43)$, respectively.

Definition 3.1. Let $V \in V_{0},(t, \mathbf{x}) \in(n \tau,(n+1) \tau] \times \mathbb{R}_{+}^{2}$. The upper right derivatives of $V(t, \mathbf{x})$ with respect to the impulsive differential system (1.4) are defined as

$$
D^{+} V(t, \mathbf{x})=\limsup _{h \rightarrow 0+} \frac{1}{h}[V(t+h, \mathbf{x}+h f(t, \mathbf{x}))-V(t, \mathbf{x})]
$$

Remark 3.2. (1) The solution of system (1.4) is a piecewise continuous function $\mathbf{x}: \mathbb{R}_{+} \rightarrow \mathbb{R}_{+}^{2}$; that is, $\mathbf{x}(t)$ is continuous on $(n \tau,(n+1) \tau], n \in \mathbb{N}$, and $\mathbf{x}\left(n \tau^{+}\right)=\lim _{t \rightarrow n \tau^{+}} \mathbf{x}(t)$ exists. (2) The smoothness properties of $f$ guarantee the global existence and uniqueness of the solutions of system (1.4) (see [22] for the details).

Definition 3.3. System (1.3) is said to be permanent if there exist positive constants $m, M$, and $T_{0}$ such that every positive solution $(x(t), y(t))$ of system (1.4) with $x_{0}, y_{0}>0$ satisfies $m \leq x(t) \leq M$ and $m \leq y(t) \leq M$ for $t>T_{0}$.

We will use the following important comparison theorem on an impulsive differential equation [22].

Lemma 3.4 (see [22]). Suppose $V \in V_{0}$ and

$$
\begin{gathered}
D^{+} V(t, \mathbf{x}) \leq g(t, V(t, \mathbf{x})), \quad t \neq n \tau, \\
V\left(t, \mathbf{x}\left(t^{+}\right)\right) \leq \psi_{n}(V(t, \mathbf{x})), \quad t=n \tau,
\end{gathered}
$$

then $g: \mathbb{R}_{+} \times \mathbb{R}_{+} \rightarrow \mathbb{R}$ is continuous on $(n \tau,(n+1) \tau] \times \mathbb{R}_{+}$and for $u(t) \in \mathbb{R}_{+}, n \in \mathbb{N}$, $\lim _{(t, y) \rightarrow\left(n \tau^{+}, u\right)} g(t, y)=g\left(n \tau^{+}, u\right)$ exists, $\psi_{n}: \mathbb{R}_{+} \rightarrow \mathbb{R}_{+}$is nondecreasing. Let $r(t)$ be the maximal solution of the scalar impulsive differential equation

$$
\begin{gathered}
u^{\prime}(t)=g(t, u(t)), \quad t \neq n \tau, \\
u\left(t^{+}\right)=\psi_{n}(u(t)), \quad t=n \tau, \\
u\left(0^{+}\right)=u_{0},
\end{gathered}
$$


existing on $[0, \infty)$. Then $V\left(0^{+}, \mathbf{x}_{0}\right) \leq u_{0}$ implies that $V(t, \mathbf{x}(t)) \leq r(t), t \geq 0$, where $\mathbf{x}(t)$ is any solution of (3.2).

We now indicate a special case of Lemma 3.4 which provides estimations for the solution of impulsive differential inequalities. For this, we let $\mathrm{PC}\left(\mathbb{R}_{+}, \mathbb{R}\right)\left(\mathrm{PC}^{1}\left(\mathbb{R}_{+}, \mathbb{R}\right)\right)$ denote the class of real piecewise continuous (real piecewise continuously differentiable) functions defined on $\mathbb{R}_{+}$.

Lemma 3.5 (see [22]). Let the function $u(t) \in P C^{1}\left(\mathbb{R}_{+}, \mathbb{R}\right)$ satisfy the inequalities

$$
\begin{gathered}
u^{\prime}(t) \leq f(t) u(t)+h(t), \quad t \neq \tau_{k}, \quad t>0 \\
u\left(\tau_{k}^{+}\right) \leq \alpha_{k} u\left(\tau_{k}\right)+\theta_{k}, \quad k \geq 0 \\
u\left(0^{+}\right) \leq u_{0}
\end{gathered}
$$

where $f, h \in P C\left(\mathbb{R}_{+}, \mathbb{R}\right)$ and $\alpha_{k} \geq 0, \theta_{k}$, and $u_{0}$ are constants and $\left(\tau_{k}\right)_{k \geq 0}$ is a strictly increasing sequence of positive real numbers. Then, for $t>0$,

$$
\begin{aligned}
u(t) \leq & u_{0}\left(\prod_{0<\tau_{k}<t} \alpha_{k}\right) \exp \left(\int_{0}^{t} f(s) d s\right)+\int_{0}^{t}\left(\prod_{s \leq \tau_{k}<t} \alpha_{k}\right) \exp \left(\int_{s}^{t} f(\gamma) d \gamma\right) h(s) d s \\
& +\sum_{0<\tau_{k}<t}\left(\prod_{\tau_{k}<\tau_{j}<t} \alpha_{j}\right) \exp \left(\int_{\tau_{k}}^{t} f(\gamma) d \gamma\right) \theta_{k} .
\end{aligned}
$$

Similar result can be obtained when all conditions of the inequalities in the Lemmas 3.4 and 3.5 are reversed.

Using Lemma 3.5, it is easy to prove that the solutions of system (1.4) with strictly positive initial value remain strictly positive as follows.

Lemma 3.6. The positive quadrant $\left(\mathbb{R}_{+}^{*}\right)^{2}$ is an invariant region for system (1.4).

Proof. Let $(x(t), y(t)):\left[0, t_{0}\right) \rightarrow \mathbb{R}^{2}$ be a solution of system (1.4) with a strictly positive initial value $\left(x_{0}, y_{0}\right)$. By Lemma 3.5, we can obtain that, for $0 \leq t<t_{0}$,

$$
\begin{aligned}
& x(t) \geq x_{0}\left(1-p_{1}\right)^{[t / \tau]} \exp \left(\int_{0}^{t} g_{1}(s) d s\right), \\
& y(t) \geq y_{0}\left(1-p_{2}\right)^{[t / \tau]} \exp \left(\int_{0}^{t} g_{2}(s) d s\right),
\end{aligned}
$$

where $g_{1}(s)=r(1-x(s) / K)-(a / c) y(s)-\lambda$ and $g_{2}(s)=-d$. Thus, $x(t)$ and $y(t)$ remain strictly positive on $\left[0, t_{0}\right)$. 
Now, we give the basic properties of the following impulsive differential equation considered the absence of the prey:

$$
\begin{gathered}
y^{\prime}(t)=-d y(t), \quad t \neq n \tau, \\
y\left(t^{+}\right)=\left(1-p_{2}\right) y(t)+q, \quad t=n \tau \\
y\left(0^{+}\right)=y_{0} .
\end{gathered}
$$

Solving the first equation of (3.7) between pulses implies

$$
y(t)=y\left(n \tau^{+}\right) \exp (-d(t-n \tau)), \quad t \in(n \tau,(n+1) \tau] .
$$

Substituting it in the second equation of (3.7), the following difference equation is obtained:

$$
y((n+1) \tau)=\left(\left(1-p_{2}\right) y(n \tau)+q\right) \exp (-d \tau)
$$

Then a periodic solution $y^{*}(t)$ of (3.7) is given by

$$
y^{*}(t)=\frac{q \exp (-d(t-n \tau))}{1-\left(1-p_{2}\right) \exp (-d \tau)}, \quad t \in(n \tau,(n+1) \tau], n \in \mathbb{N}
$$

Thus we can easy obtain the following results.

Lemma 3.7. (1) $y^{*}(t)=q \exp (-d(t-n \tau)) /\left(1-\left(1-p_{2}\right) \exp (-d \tau)\right), t \in(n \tau,(n+1) \tau], n \in \mathbb{N}$, and $y^{*}\left(0^{+}\right)=q /\left(1-\left(1-p_{2}\right) \exp (-d \tau)\right)$ is a positive periodic solution of (3.7).

(2) $y(t)=\left(1-p_{2}\right)^{n+1}\left(y_{0}-q \exp (-d \tau) /\left(1-\left(1-p_{2}\right) \exp (-d \tau)\right)\right) \exp (-d \tau)+y^{*}(t)$ is a general solution of (3.7) with $y_{0} \geq 0, t \in(n \tau,(n+1) \tau]$ and $n \in \mathbb{N}$.

(3) For every solution $y(t)$ and every positive periodic solution $y^{*}(t)$ of system (3.7), it follows that $y(t)$ tends to $y^{*}(t)$ as $t \rightarrow \infty$. Thus, the complete expression for the prey-free periodic solution of system (1.4) is obtained $\left(0, y^{*}(t)\right)=\left(0, q \exp (-d(t-n \tau)) /\left(1-\left(1-p_{2}\right) \exp (-d \tau)\right)\right)$ for $t \in$ $(n \tau,(n+1) \tau]$.

Now, we discuss the stability of the prey-free periodic solution $\left(0, y^{*}(t)\right)$.

Theorem 3.8. (1) The prey-free periodic solution $\left(0, y^{*}(t)\right)$ of system (1.4) is locally asymptotically stable if

$$
(r+\lambda) \tau+\frac{a}{b d} \ln \frac{b q \exp (-d \tau)+c\left(1-\left(1-p_{2}\right) \exp (-d \tau)\right)}{b q+c\left(1-\left(1-p_{2}\right) \exp (-d \tau)\right)}<\ln \frac{1}{1-p_{1}}
$$

(2) Moreover, $\left(0, y^{*}(t)\right)$ is globally asymptotically stable if

$$
(r+\lambda) \tau+\frac{a}{b d} \ln \frac{r b q \exp (-d \tau)+(K(r+\lambda)+r c)\left(1-\left(1-p_{2}\right) \exp (-d \tau)\right)}{r b q+(K(r+\lambda)+r c)\left(1-\left(1-p_{2}\right) \exp (-d \tau)\right)}<\ln \frac{1}{1-p_{1}}
$$


Proof. To show the local stability of the prey-free periodic solution $\left(0, y^{*}(t)\right)$ of system (1.4), consider the following impulsive differential system:

$$
\begin{gathered}
x_{1}^{\prime}(t)=r x_{1}(t)\left(1-\frac{x_{1}(t)}{K}\right)-\frac{a x_{1}(t) y_{1}(t)}{b y_{1}(t)+x_{1}(t)+c}+\lambda x_{1}(t), \quad t \neq n \tau, \\
y_{1}^{\prime}(t)=-d y_{1}(t)+\frac{e a x_{1}(t) y_{1}(t)}{b y_{1}(t)+x_{1}(t)+c}, \\
x_{1}\left(t^{+}\right)=\left(1-p_{1}\right) x_{1}(t), \quad t=n \tau, \\
y_{1}\left(t^{+}\right)=\left(1-p_{2}\right) y_{1}(t)+q, \\
\left(x_{1}\left(0^{+}\right), y_{1}\left(0^{+}\right)\right)=\left(x_{0}, y_{0}\right) .
\end{gathered}
$$

By Lemma 3.4, $x(t) \leq x_{1}(t)$ and $y(t) \leq y_{1}(t)$, where $(x(t), y(t))$ is a solution of system (1.4). Note that if $\left(x_{1}(t), y_{1}(t)\right)$ is locally stable, then so is $(x(t), y(t))$. It is easy to see that the periodic solution $\left(0, y_{1}^{*}(t)\right)$ of $(3.13)$ is the same as that of $(1.4)$. That is, $y_{1}^{*}(t)=y^{*}(t)$. The local stability of the periodic solution $\left(0, y_{1}^{*}(t)\right)$ may be determined by considering the behavior of small amplitude perturbations of the solution. Define $x_{1}(t)=u(t), y_{1}(t)=y_{1}^{*}(t)+v(t)$. Then they may be written as

$$
\left(\begin{array}{l}
u(t) \\
v(t)
\end{array}\right)=\Phi(t)\left(\begin{array}{l}
u(0) \\
v(0)
\end{array}\right), \quad 0 \leq t \leq \tau,
$$

where $\Phi(t)$ satisfies

$$
\frac{d \Phi}{d t}=\left(\begin{array}{cc}
r+\lambda-\frac{a y_{1}^{*}(t)}{b y_{1}^{*}(t)+c} & 0 \\
\frac{e a y_{1}^{*}(t)}{b y_{1}^{*}(t)+c} & -d
\end{array}\right) \Phi(t),
$$

and $\Phi(0)=I$, where $I$ is the identity matrix. The linearization of the third and fourth equations of system (1.4) becomes

$$
\left(\begin{array}{l}
u\left(n \tau^{+}\right) \\
v\left(n \tau^{+}\right)
\end{array}\right)=\left(\begin{array}{cc}
1-p_{1} & 0 \\
0 & 1-p_{2}
\end{array}\right)\left(\begin{array}{l}
u(n \tau) \\
v(n \tau)
\end{array}\right) .
$$

Note that all eigenvalues of

$$
\left(\begin{array}{cc}
1-p_{1} & 0 \\
0 & 1-p_{2}
\end{array}\right) \Phi(\tau)
$$

are $\mu_{1}=\left(1-p_{1}\right) \exp \left(\int_{0}^{\tau} r+\lambda-a y_{1}^{*}(t) /\left(b y_{1}^{*}(t)+c\right) d t\right)$ and $\mu_{2}=\left(1-p_{2}\right) \exp (-d \tau)<1$. 
Since $\int_{0}^{\tau} a y_{1}^{*}(t) /\left(b y_{1}^{*}(t)+c\right) d t=-(a / b d) \ln \left(\left(b q \exp (-d \tau)+c\left(1-\left(1-p_{2}\right) \exp (-d \tau)\right)\right) /(b q+\right.$ $\left.\left.c\left(1-\left(1-p_{2}\right) \exp (-d \tau)\right)\right)\right)$, the condition $\left|\mu_{2}\right|<1$ is equivalent to (3.11). According to Floquet theory [22], $\left(0, y^{*}(t)\right)=\left(0, y_{1}^{*}(t)\right)$ is locally asymptotically stable.

It is easy to see that the solution $\left(0, y^{*}(t)\right)$ is locally stable if condition (3.12) holds. Now, to prove the global stability of the pest-free periodic solution, let $(x(t), y(t))$ be a solution of system (1.4). From (3.12), we can select a sufficiently small number $\epsilon_{1}>0$ satisfying

$$
\rho=\left(1-p_{1}\right) \exp \left(\int_{0}^{\tau} r+\lambda-\frac{r a\left(y^{*}(t)-\epsilon_{1}\right)}{r b\left(y^{*}(t)-\epsilon_{1}\right)+K(r+\lambda)+r \epsilon_{1}+r c} d t\right)<1 .
$$

It follows from the first equation in (1.4) that $x^{\prime}(t) \leq x(t)(r+\lambda-(r / K) x(t))$ for $t \neq n \tau$. From Lemma 3.4, we have $x(t) \leq u(t)$, where $u(t)$ is a solution of the following impulsive differential equation:

$$
\begin{gathered}
u^{\prime}(t)=u(t)\left(r+\lambda-\frac{r}{K} u(t)\right), \quad t \neq n \tau, \\
u\left(t^{+}\right)=\left(1-p_{1}\right) u(t), \quad t=n \tau \\
u\left(0^{+}\right)=x_{0} .
\end{gathered}
$$

Since $u(t) \rightarrow K(r+\lambda) / r$ as $t \rightarrow \infty$ if $x_{0}>0, x(t) \leq K(r+\lambda) / r+\epsilon$ for any $\epsilon>0$ with $t$ large enough. For simplicity, we may assume that $x(t) \leq K(r+\lambda) / r+\epsilon_{1}$ for all $t>0$. Since $y^{\prime}(t) \geq-d y(t)$, it follow from Lemma 3.4 that $y(t) \geq v(t)>y^{*}(t)-\epsilon_{1}$ for $t$ sufficiently large, where $v(t)$ is a solution of the following impulsive differential equation:

$$
\begin{gathered}
v^{\prime}(t)=-d v(t), \quad t \neq n \tau, \\
v\left(t^{+}\right)=\left(1-p_{2}\right) v(t)+q, \quad t=n \tau, \\
v\left(0^{+}\right)=y_{0} .
\end{gathered}
$$

For simplicity, we may suppose that $y(t) \geq v(t)>y^{*}(t)-\epsilon_{1}$ for all $t \geq 0$. From system (1.4), we obtain

$$
\begin{gathered}
x^{\prime}(t) \leq x(t)\left(r+\lambda-\frac{a\left(y^{*}(t)-\epsilon_{1}\right)}{b\left(y^{*}(t)-\epsilon_{1}\right)+K(r+\lambda) / r+\epsilon_{1}+c}\right), \quad t \neq n \tau, \\
x\left(t^{+}\right)=\left(1-p_{1}\right) x(t), \quad t=n \tau .
\end{gathered}
$$

Integrating (3.21) on $(n \tau,(n+1) \tau]$, we get

$$
\begin{aligned}
x((n+1) \tau) & \leq\left(1-p_{1}\right) x(n \tau) \exp \left(\int_{n \tau}^{(n+1) \tau} r+\lambda-\frac{r a\left(y^{*}(t)-\epsilon_{1}\right)}{r b\left(y^{*}(t)-\epsilon_{1}\right)+K(r+\lambda)+r \epsilon_{1}+r c} d t\right) \\
& =x(n \tau) \rho
\end{aligned}
$$


and hence $x((n+1) \tau) \leq x(n \tau) \rho^{n}$ which implies that $x(n \tau) \rightarrow 0$ as $n \rightarrow \infty$. Further, we obtain, for $t \in(n \tau,(n+1) \tau]$,

$$
\begin{aligned}
x(t) & \leq\left(1-p_{1}\right) x(n \tau) \exp \left(\int_{n \tau}^{t} r+\lambda-\frac{r a\left(y^{*}(t)-\epsilon_{1}\right)}{r b\left(y^{*}(t)-\epsilon_{1}\right)+K(r+\lambda)+r \epsilon_{1}+r c} d t\right) \\
& \leq x(n \tau) \exp \left(\left(r+\lambda+\frac{a}{c} \epsilon_{1}\right) \tau\right)
\end{aligned}
$$

which implies that $x(t) \rightarrow 0$ as $t \rightarrow \infty$. Now, take a sufficiently small number $\epsilon_{2}>0$ satisfying $\epsilon_{2}<d$. Since $\lim _{t \rightarrow \infty} x(t)=0$, we may assume that $x(t) \leq \epsilon_{2}$ for all $t \geq 0$. It follows from the second equation in (1.4) that, for $t \neq n \tau$,

$$
y^{\prime}(t) \leq y(t)\left(-d+\frac{e a}{c} \epsilon_{2}\right)
$$

Thus, by Lemma 3.4, we induce that $y(t) \leq \tilde{y}^{*}(t)$, where $\tilde{y}^{*}(t)$ is the periodic solution of (3.7) with $d$ changed into $d-(e a / c) \epsilon_{2}$. By taking sufficiently small $\epsilon_{1}$ and $\epsilon_{2}$, we obtain that $y(t)$ tends to $y^{*}(t)$ as $t \rightarrow \infty$. theorems.

Using the similar method to the proof of Theorem 3.8, we obtain the following

Theorem 3.9. For system (1.5), the periodic solution $\left(0, y^{*}(t)\right)$ is locally asymptotically stable if $(r+\lambda) \tau-a q(1-\exp (-d \tau)) / c d\left(1-\left(1-p_{2}\right) \exp (-d \tau)\right)<\ln \left(1 /\left(1-p_{1}\right)\right)$, and moreover, it is globally asymptotically stable if $(r+\lambda) \tau-r a q(1-\exp (-d \tau)) / d(K(r+\lambda)+r c)\left(1-\left(1-p_{2}\right) \exp (-d \tau)\right)<$ $\ln \left(1 /\left(1-p_{1}\right)\right)$.

Theorem 3.10. For system (1.6), the periodic solution $\left(0, y^{*}(t)\right)$ is locally asymptotically stable if $(r+\lambda-a / b) \tau<\ln \left(1 /\left(1-p_{1}\right)\right)$, and moreover, it is globally asymptotically stable if $(r+$ $\lambda) \tau+(a / b d) \ln \left(\left(r b q \exp (-d \tau)+K(r+\lambda)\left(1-\left(1-p_{2}\right) \exp (-d \tau)\right)\right) /(r b+K(r+\lambda)(1-(1-\right.$ $\left.\left.\left.\left.p_{2}\right) \exp (-d \tau)\right)\right)\right)<\ln \left(1 /\left(1-p_{1}\right)\right)$.

Now, we prove the boundedness of system (1.4).

Theorem 3.11. There is an $M>0$ such that $x(t), y(t) \leq M$ for all t large enough, where $(x(t), y(t))$ is a solution of system (1.4).

Proof. Let $\mathbf{x}(t)=(x(t), y(t))$ be a solution of system (1.4) and let $V(t, \mathbf{x})=e x(t)+y(t)$. Then $V \in V_{0}$. If $t \neq n \tau$, then we obtain

$$
D^{+} V+\beta V=-\frac{e r}{K} x(t)^{2}+e(r+\lambda \sin (\omega t)+\beta) x(t)+(\beta-d) y(t)
$$

$V\left(n \tau^{+}\right) \leq V(n \tau)+q$ and $V\left(0^{+}\right)=e x_{0}+y_{0}$. Clearly, the right-hand side of (3.25) is bounded by a constant $M_{0}>0$ if $0<\beta<d$. So we can choose $\beta_{0}$ such that

$$
\begin{gathered}
D^{+} V \leq-\beta_{0} V+M_{0}, \quad t \neq n \tau \\
V\left(n \tau^{+}\right) \leq V(n \tau)+q .
\end{gathered}
$$


From Lemma 3.4, we obtain that

$$
V(t) \leq\left(V\left(0^{+}\right)-\frac{M_{0}}{\beta_{0}}\right) \exp \left(-\beta_{0} t\right)+\frac{q\left(1-\exp \left(-(n+1) \beta_{0} \tau\right)\right)}{1-\exp \left(-\beta_{0} \tau\right)} \exp \left(-\beta_{0}(t-n \tau)\right)+\frac{M_{0}}{\beta_{0}}
$$

for $t \in(n \tau,(n+1) \tau]$. Therefore, $V(t)$ is bounded by a constant $M>0$ for sufficiently large $t$. Hence $x(t) \leq M, y(t) \leq M$ for a solution $(x(t), y(t))$ with all $t$ large enough.

The boundedness of systems (1.5) and (1.6) can be obtained from Theorem 3.11.

Next, we investigate the permanence of system (1.4).

Theorem 3.12. System (1.4) is permanent if

$$
(r-\lambda) \tau+\frac{a}{b d} \ln \frac{b q \exp (-d \tau)+c\left(1-\left(1-p_{2}\right) \exp (-d \tau)\right)}{b q+c\left(1-\left(1-p_{2}\right) \exp (-d \tau)\right)}>\ln \frac{1}{1-p_{1}}
$$

Proof. Let $x_{0}, y_{0}>0$. Consider the following system:

$$
\begin{gathered}
x_{2}^{\prime}(t)=r x_{2}(t)\left(1-\frac{x_{2}(t)}{K}\right)-\frac{a x_{2}(t) y_{2}(t)}{b y_{2}(t)+x_{2}(t)+c}-\lambda x_{2}(t), \quad t \neq n \tau, \\
y_{2}^{\prime}(t)=-d y_{2}(t)+\frac{e a x_{2}(t) y_{2}(t)}{b y_{2}(t)+x_{2}(t)+c^{\prime}} \\
x_{2}\left(t^{+}\right)=\left(1-p_{1}\right) x_{2}(t), \quad t=n \tau, \\
y_{2}\left(t^{+}\right)=\left(1-p_{2}\right) y_{2}(t)+q, \\
\left(x_{2}\left(0^{+}\right), y_{2}\left(0^{+}\right)\right)=\left(x_{0}, y_{0}\right) .
\end{gathered}
$$

It follows from Lemma 3.4 that $x(t) \geq x_{2}(t)$ and $y(t) \geq y_{2}(t)$. From Theorem 3.11, we may assume that $x_{2}(t)<M, y_{2}(t)<M$, for all $t \geq 0$ large enough and $M>(r-\lambda) / a$. Let $m_{2}=q \exp (-d \tau) /\left(1-\left(1-p_{2}\right) \exp (-d \tau)\right)-\epsilon_{2}, \epsilon_{2}>0$. From Lemmas 3.4 and 3.7, we obtain $y_{2}(t) \geq m_{2}$ for all $t$ large enough. Thus we will show that $x_{2}(t)$ has a lower bound $m_{1}>0$ for all $t$ large enough. We will do this in the following two steps.

Step 1. From (3.28), we can choose $m_{3}>0, \epsilon_{1}>0$ small enough such that $\delta<d$ and $\eta=$ $\left(1-p_{1}\right) \exp \left(\left(r-\mathcal{\lambda}-(r / K) m_{3}\right) \tau+(a / b d) \ln (A / B)-\left(a \epsilon_{1} / c\right) \tau\right)>1$, where $\delta=e a m_{3} / c, A=$ $b q \exp (-d \tau)+c\left(1-\left(1-p_{2}\right) \exp (-d \tau)\right)$ and $B=b q+c\left(1-\left(1-p_{2}\right) \exp (-d \tau)\right)$. Suppose that $x_{2}(t)<m_{3}$ for all $t$. Then, from the second equation of system (3.29), we obtain $y_{2}^{\prime}(t) \leq$ $y_{2}(t)\left(-d+(e a / c) x_{2}(t)\right) \leq y_{2}(t)(-d+\delta)$. By Lemmas 3.4 and 3.7, we get $y_{2}(t) \leq u(t)$ and $u(t) \rightarrow u^{*}(t)$ as $t \rightarrow \infty$, where $u(t)$ is the solution of

$$
\begin{gathered}
u^{\prime}(t)=(-d+\delta) u(t), \quad t \neq n \tau, \\
u\left(t^{+}\right)=\left(1-p_{2}\right) u(t)+q, \quad t=n \tau, \\
u\left(0^{+}\right)=u_{0}>0,
\end{gathered}
$$




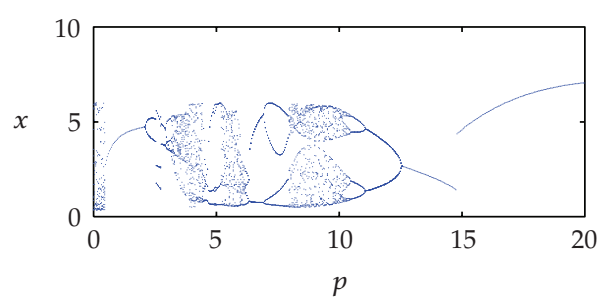

(a)

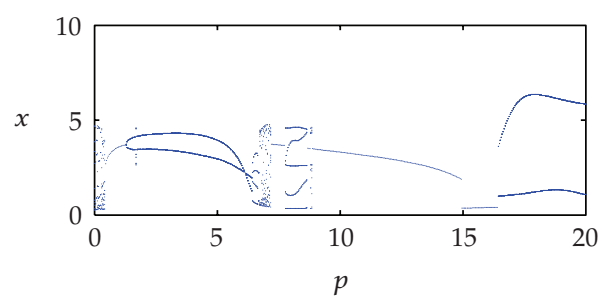

(c)

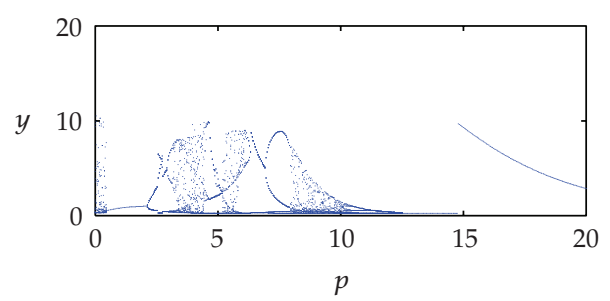

(b)

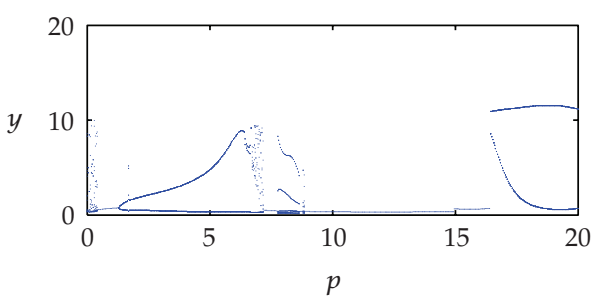

(d)

Figure 5: Bifurcation diagrams of system (1.4). (a)-(b) $x, y$ are plotted for $p$ when $\lambda=0$, (c)-(d) $x, y$ are plotted for $p$ when $\lambda=1$.

and $u^{*}(t)=q \exp ((-d+\delta)(t-n \tau)) /\left(1-\left(1-p_{2}\right) \exp ((-d+\delta) \tau)\right), t \in(n \tau,(n+1) \tau]$. Then there exists $T_{1}>0$ such that $y_{2}(t) \leq u(t) \leq u^{*}(t)+\epsilon_{1}$ for $t \geq T_{1}$. So, if $t \neq n \tau, t \geq T_{1}$, then

$$
\begin{aligned}
x_{2}^{\prime}(t) & \geq x_{2}(t)\left(r-\lambda-\frac{r}{K} m_{3}-\frac{a u^{*}(t)+a \epsilon_{1}}{b u^{*}(t)+b \epsilon_{1}+c}\right) \\
& \geq x_{2}(t)\left(r-\lambda-\frac{r}{K} m_{3}-\frac{a u^{*}(t)}{b u^{*}(t)+c}-\frac{a \epsilon_{1}}{c}\right),
\end{aligned}
$$

and if $t=n \tau, t \geq T_{1}$, then $x\left(t^{+}\right)=\left(1-p_{1}\right) x(t)$. Let $N_{1} \in \mathbb{N}$ and $N_{1} \tau \geq T_{1}$. Integrating (3.31) on $(n \tau,(n+1) \tau], n \geq N_{1}$, we have $x_{2}((n+1) \tau) \geq\left(1-p_{1}\right) x_{2}(n \tau) \exp \left(\int_{n \tau}^{(n+1) \tau} r-\lambda-(r / K) m_{3}-\right.$ $\left.a u^{*}(t) /\left(b u^{*}(t)+c\right)-\left(a \epsilon_{1} / c\right) d t\right) \geq x_{2}(n \tau) \exp (\eta)$. Then $x_{2}\left(\left(N_{1}+k\right) \tau\right) \geq x_{2}\left(N_{1} \tau\right) \exp (k \eta) \rightarrow \infty$ as $k \rightarrow \infty$ which is a contradiction to the boundedness of $x_{2}(t)$. Hence there exists a $t_{1}>0$ such that $x_{2}\left(t_{1}\right) \geq m_{3}$.

Step 2. If $x_{2}(t) \geq m_{3}$ for all $t \geq t_{1}$, then we are done. If not, we may let $t^{*}=\inf _{t>t_{1}}\left\{x_{2}(t)<m_{3}\right\}$. Then $x_{2}(t) \geq m_{3}$ for $t \in\left[t_{1}, t^{*}\right)$ and, by the continuity of $x_{2}(t)$, we have $x_{2}\left(t^{*}\right)=m_{3}$. Suppose that $t^{*} \in\left[n_{1} \tau,\left(n_{1}+1\right) \tau\right)$ for some $n_{1} \in \mathbb{N}$. Select $n_{2}, n_{3} \in \mathbb{N}$ such that $n_{2} \tau(\delta-d)<\ln \left(\epsilon_{1} /(M+p)\right)$ and $\left(1-p_{1}\right)^{n_{2}} \exp \left(\left(n_{2}+1\right) \eta_{1} \tau\right) \exp \left(n_{3} \eta\right)>1$, where $\eta_{1}=r-\lambda-(r / K) m_{3}-a M<0$. Let $T=n_{2} \tau+n_{3} \tau$. Then we have only to consider two possible cases for $t \in\left(t^{*},\left(n_{1}+1\right) \tau\right]$.

Case $1\left(x_{2}(t)<m_{3}\right.$ for $\left.t \in\left(t^{*},\left(n_{1}+1\right) \tau\right]\right)$. In this case we will show that there exists $t_{2} \in$ $\left[\left(n_{1}+1\right) \tau,\left(n_{1}+1\right) \tau+T\right]$ such that $x_{2}\left(t_{2}\right) \geq m_{3}$. Suppose not, that is, $x_{2}(t)<m_{3}, t \in\left[\left(n_{1}+\right.\right.$ 1) $\left.\tau,\left(n_{1}+1+n_{2}+n_{3}\right) \tau\right]$. Then $x_{2}(t)<m_{3}$ for all $t \in\left(t^{*},\left(n_{1}+1+n_{2}+n_{3}\right) \tau\right]$. By (3.30) with 


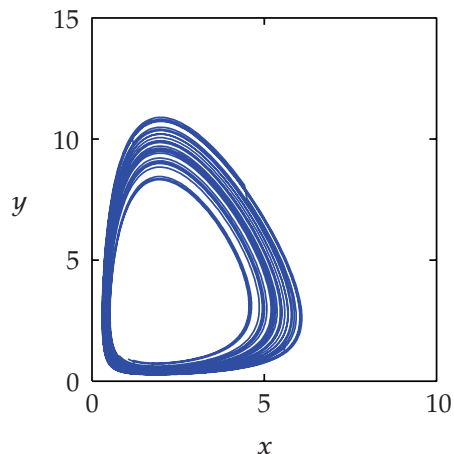

(a)

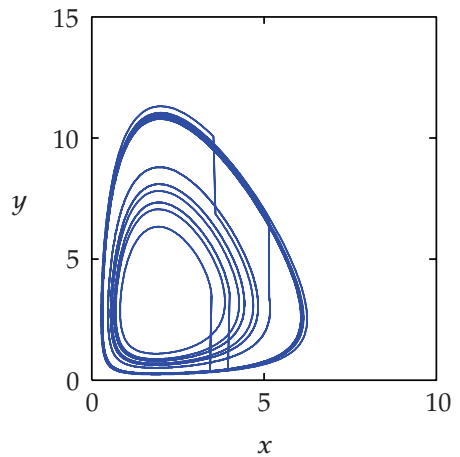

(b)

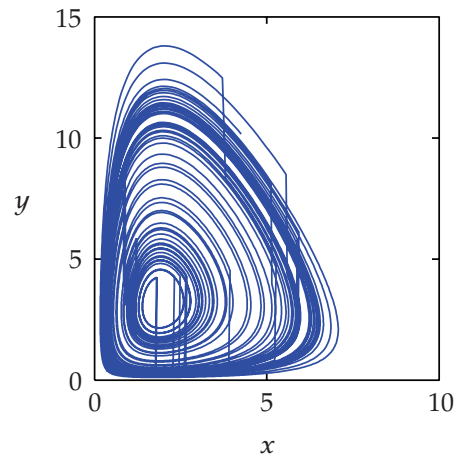

(c)

Figure 6: Phase portraits of solutions for system (1.4) when $\lambda=0$. (a) $p=0.4$, (b) $p=3.1$ (a $4 \tau$-periodic solution), (c) $p=4$.

$u\left(\left(n_{1}+1\right) \tau^{+}\right)=y_{2}\left(\left(n_{1}+1\right) \tau^{+}\right)$, we obtain

$$
\begin{aligned}
u(t)= & \left(1-p_{1}\right)^{n_{1}+1}\left(u\left(\left(n_{1}+1\right) \tau^{+}\right)-\frac{p}{1-\left(1-p_{2}\right) \exp (-d+\delta)}\right) \\
& \times \exp \left((-d+\delta)\left(t-\left(n_{1}+1\right) \tau\right)\right)+u^{*}(t)
\end{aligned}
$$

for $t \in(n \tau,(n+1) \tau], n_{1}+1 \leq n \leq n_{1}+n_{2}+n_{3}$. So we get $\left|u(t)-u^{*}(t)\right| \leq(M+p) \exp \left((-d+\delta) n_{2} \tau\right)<$ $\epsilon_{1}$ and $y_{2}(t) \leq u(t) \leq u^{*}(t)+\epsilon_{1}$ for $t \in\left[\left(n_{1}+1+n_{2}\right) \tau,\left(n_{1}+1+n_{2}+n_{3}\right) \tau\right]$. Also we obtain that $x_{2}^{\prime}(t) \geq x_{2}(t)\left(r-\lambda-(r / K) m_{3}-\left(a u^{*}(t) /\left(b u^{*}(t)+c\right)-a \epsilon_{1} / c\right)\right.$ if $t \neq n \tau$ and $x_{2}\left(t^{+}\right)=\left(1-p_{1}\right) x_{2}(t)$ if $t=n \tau$, for $t \in\left[\left(n_{1}+1+n_{2}\right) \tau,\left(n_{1}+1+n_{2}+n_{3}\right) \tau\right]$. Similarly to Step 1 , we have

$$
x_{2}\left(\left(n_{1}+1+n_{2}+n_{3}\right) \tau\right) \geq x_{2}\left(\left(n_{1}+1+n_{2}\right) \tau\right) \exp \left(n_{3} \eta\right) .
$$

Since (3.29) and $y_{2}(t) \leq M$ we have, for all $t \in\left(t^{*},\left(n_{1}+1+n_{2}\right) \tau\right], x_{2}^{\prime}(t) \geq x_{2}(t)\left(r-\lambda-(r / K) m_{3}-\right.$ $a M)=\eta_{1} x_{2}(t)$ if $t \neq n \tau$ and $x_{2}\left(t^{+}\right)=\left(1-p_{1}\right) x_{2}(t)$ if $t=n \tau$. Integrating it on $\left[t^{*},\left(n_{1}+1+n_{2}\right) \tau\right]$ we obtain that

$$
\begin{aligned}
x_{2}\left(\left(n_{1}+1+n_{2}\right) \tau\right) & \geq m_{3}\left(1-p_{1}\right)^{n_{2}} \exp \left(\eta_{1}\left(\left(n_{1}+1+n_{2}\right) \tau-t^{*}\right)\right) \\
& \geq m_{3}\left(1-p_{1}\right)^{n_{2}} \exp \left(\eta_{1}\left(n_{2}+1\right) \tau\right) .
\end{aligned}
$$

Thus $x_{2}\left(\left(n_{1}+1+n_{2}+n_{3}\right) \tau\right) \geq m_{3}\left(1-p_{1}\right)^{n_{2}} \exp \left(\eta_{1}\left(n_{2}+1\right) \tau\right) \exp \left(n_{3} \eta\right)>m_{3}$ which is a contradiction.

Now, let $\bar{t}=\inf _{t>t^{*}}\left\{x_{2}(t) \geq m_{3}\right\}$. Then $x_{2}(t) \leq m_{3}$ for $t^{*} \leq t<\bar{t}$ and $x_{2}(\bar{t})=m_{3}$. So, we have, for $t \in\left[t^{*}, \bar{t}\right), x_{2}^{\prime}(t) \geq x_{2}(t)\left(r-\lambda-(r / K) m_{3}-a M\right)$ if $t \neq n \tau$ and $x_{2}\left(t^{+}\right)=\left(1-p_{1}\right) x_{2}(t)$ if $t=n \tau$. By the integration of it on $\left[t^{*}, t\right)$ for $\left(t^{*} \leq t \leq \bar{t}\right)$, we can get that $x_{2}(t) \geq x_{2}\left(t^{*}\right)(1-$ $\left.p_{1}\right)^{1+n_{1}+n_{2}} \exp \left(\eta_{1}\left(t-t^{*}\right)\right) \geq m_{3}\left(1-p_{1}\right)^{1+n_{1}+n_{2}} \exp \left(\eta_{1}\left(1+n_{2}+n_{3}\right) \tau\right) \equiv m_{1}>0$. 


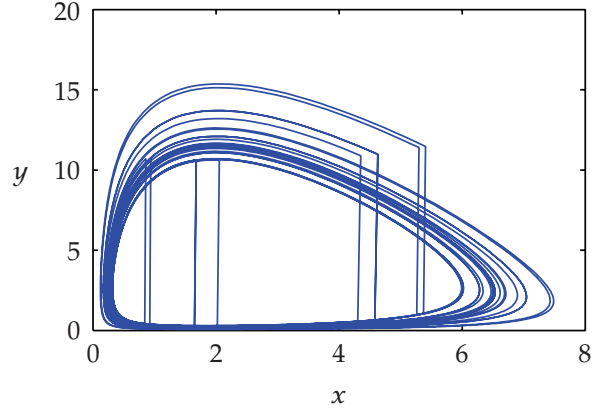

(a)

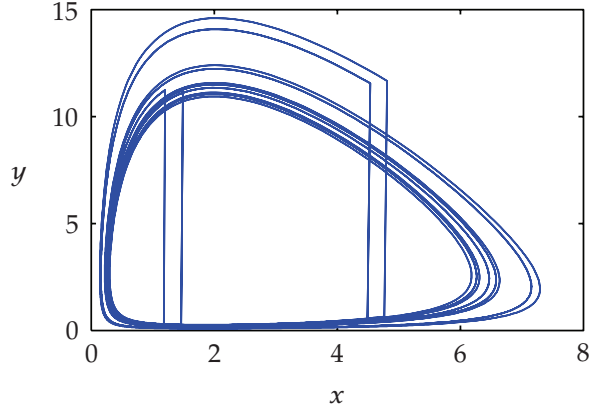

(b)

Figure 7: Phase portraits of solutions for system (1.4) when $\lambda=0$. (a) $p=10.4$ (a $8 \tau$-periodic solution), (b) $p=11$ (a $4 \tau$-periodic solution).

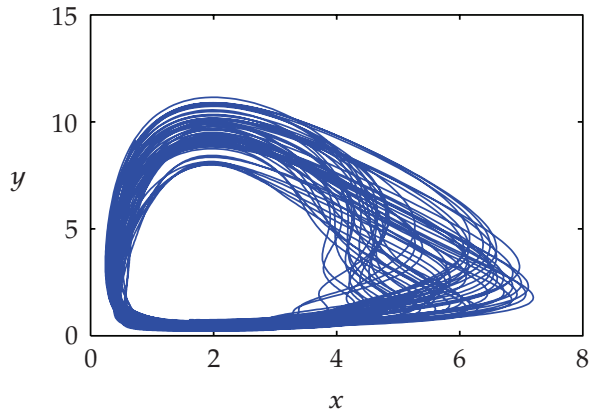

(a)

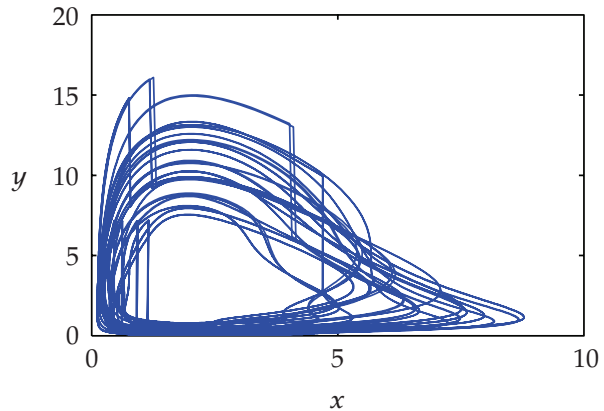

(b)

Figure 8: Phase portraits of solutions for system (1.4) when $\lambda=1$. (a) $p=0.35$, (b) $p=6.9$.

Case 2 (there is a $t^{\prime} \in\left(t^{*},\left(n_{1}+1\right) \tau\right]$ such that $\left.x_{2}\left(t^{\prime}\right) \geq m_{3}\right)$. Let $t=\inf _{t>t^{*}}\left\{x_{2}(t) \geq m_{3}\right\}$. Then $x_{2}(t) \leq m_{3}$ for $t \in\left[t^{*}, \underline{t}\right)$ and $x_{2}(\underline{t})=m_{3}$. For $t \in\left[t^{*}, \underline{t}\right), x_{2}^{\prime}(t) \geq x_{2}(t)\left(r-\lambda-(r / K) m_{3}-a M\right)$ if $n \neq n \tau$. Integrating the equation on $\left[t^{*}, \underline{t}\right)\left(t^{*} \leq \underline{t} \leq t\right)$, we can get that $x_{2}(t) \geq x_{2}\left(t^{*}\right) \exp \left(\eta_{1}(t-\right.$ $\left.\left.t^{*}\right)\right) \geq m_{3} \exp \left(\eta_{1} \tau\right) \geq m_{1}$.

Thus, in both cases the similar argument can be continued since $x_{2}(t) \geq m_{3}$ for some $t>t_{1}$. This completes the proof.

Applying the method used in the proof of Theorem 3.12 to systems (1.5) and (1.6), we obtain the following results.

Theorem 3.13. System (1.5) is permanent if $(r-\lambda) \tau-a q(1-\exp (-d \tau)) / c d\left(1-\left(1-p_{2}\right) \exp (-d \tau)\right)>$ $\ln \left(1 /\left(1-p_{1}\right)\right)$.

Theorem 3.14. System (1.6) is permanent if $(r-\lambda-a / b) \tau>\ln \left(1 /\left(1-p_{1}\right)\right)$. 


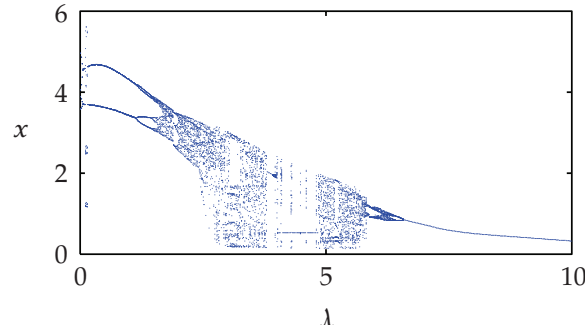

(a)

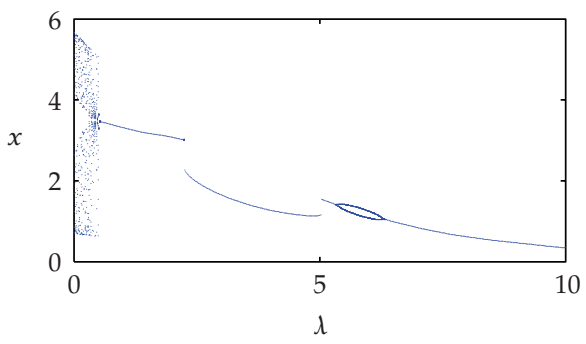

(c)

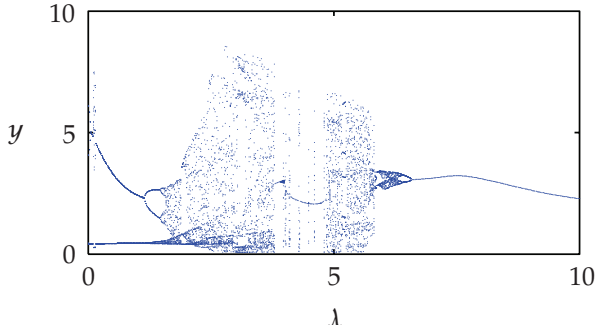

(b)

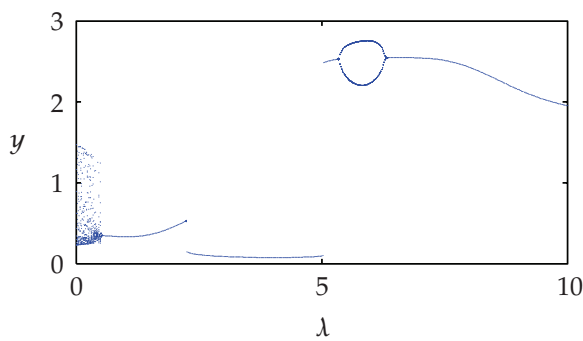

(d)

Figure 9: Bifurcation diagrams of system (1.4). (a)-(b) $x, y$ are plotted for $\lambda$ when $p=3$, (c)-(d) $x, y$ are plotted for $\lambda$ when $p=10$.

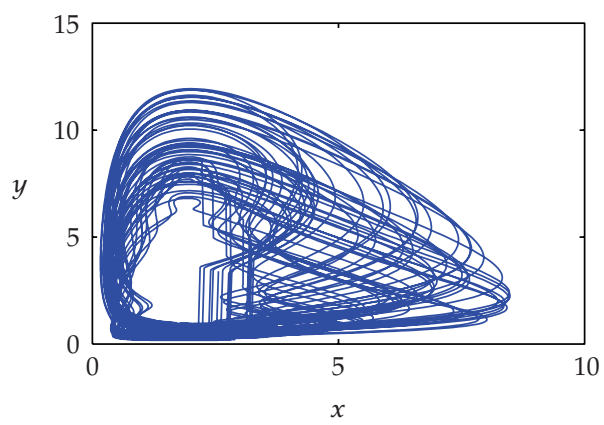

(a)

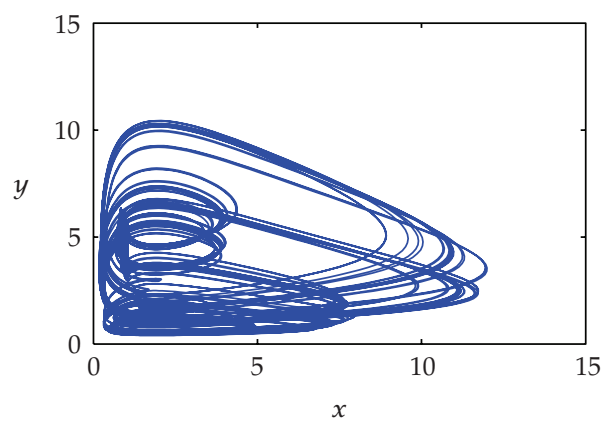

(b)

Figure 10: Phase portraits of solutions for system (1.4) when $p=3$. (a) $\lambda=2.3$, (b) $\lambda=6.2$.

\section{Numerical Analysis of Seasonal Effect and Impulsive Perturbation}

In this section we will study the influence of impulsive perturbation and seasonal effects on system (1.4), and the relationship between seasonal effects and impulsive perturbation. For this, we take the same parameters as those in Section 2, $p_{1}=p_{2}=0$ and $\tau=$ 10.

First, we display bifurcation diagrams for system (1.4) as $p$ increases from 0 to 20 about $\lambda=0$ and $\lambda=1$ in Figure 5. From Figures 5(a) and 5(b), we see that system (1.4) experiences quasiperiodic oscillation (see Figure 6(a)) when $p<p_{1}(\approx 0.5)$. However, when $p>p_{1}$, we see that there is a cascade of periodic bifurcation (see Figure $6(\mathrm{~b})$ ) 


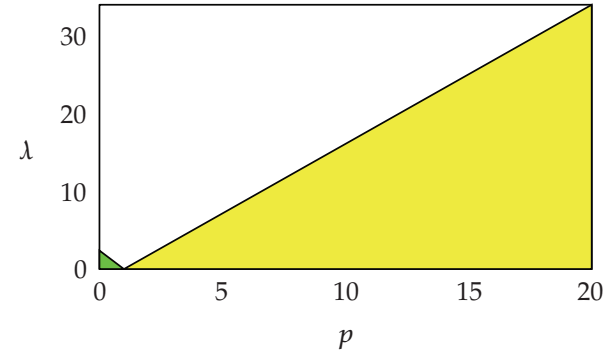

(a)

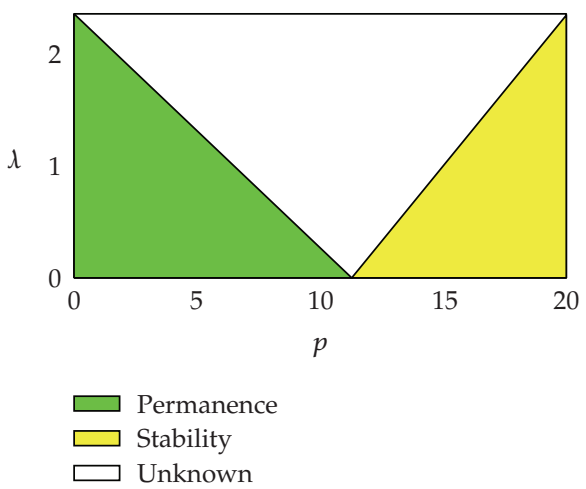

(c)

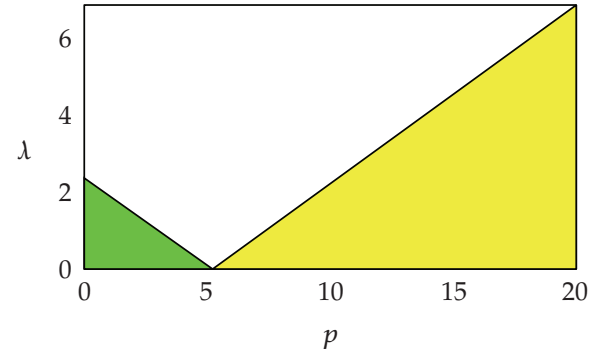

(b)

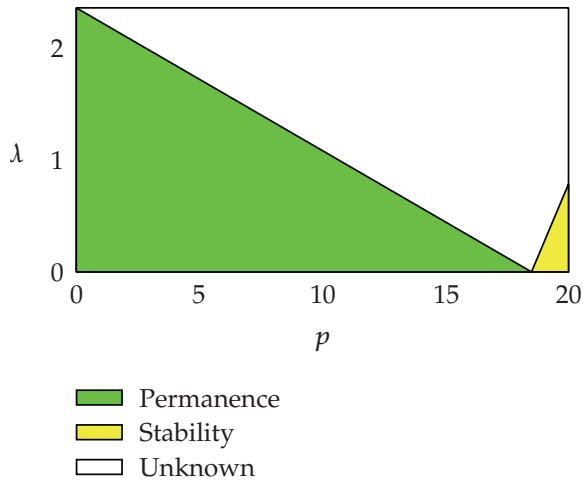

(d)

Figure 11: Regions induced from Theorems 3.8 and 3.12. (a) $\tau=0.1$, (b) $\tau=0.5$, (c) $\tau=1.5$, (d) $\tau=2$.

leading to chaos (see Figure 6(c)), which is followed by a cascade of periodic halving bifurcation from chaos to periodic solutions (see Figure 7). Figures 5(c) and 5(d) clearly show that with $p$ increasing from 0 to 20 , system (1.4) experiences process of periodic oscillating $\rightarrow$ periodic doubling $\rightarrow$ chaos $\rightarrow$ periodic halving. Figure 8 displays two different strange attractors. Next, Figure 9 illustrates bifurcation diagrams for different values of the pulse $p$ and $\lambda$ as a bifurcation parameter. It follows from Figures 9(a) and 9(b) that system (1.4) experiences process of periodic oscillating with different periods $\rightarrow$ periodic doubling $\rightarrow$ chaos $\rightarrow$ periodic windows with periodic halving cascade $\rightarrow \tau$-periodic solutions. Figure 10 exhibits two different strange attractors. It follows from Figures 9 (c) and $9(\mathrm{~d})$ that system (1.4) undergoes chaotic motions when $\lambda<\lambda_{1}(\approx 0.51)$. When $\lambda>\lambda_{1}$, chaotic motions suddenly disappear and appear as $\tau$-periodic solutions. There are also periodic doubling and halving phenomena. Finally, we investigate the relationship between $p, \lambda$, and $\tau$ in a view of controlling the population density of the prey and predator. As seen in Figure 11, we figure out that the longer the period $\tau$ is, the larger the permanence region is and the smaller the stability region is. That means that we should release the predator within a short period, or the impulsive perturbations of the predator should be occurred at short intervals, to eradicate the prey. On the contrary, the impulsive perturbations of the predator should be occurred at long-time intervals for coexistence of the prey and the predator. If we choose $(p, \lambda, \tau)=(5,1,1.5)$, we can see coexistence of the prey and predator as shown in Figure 12. 


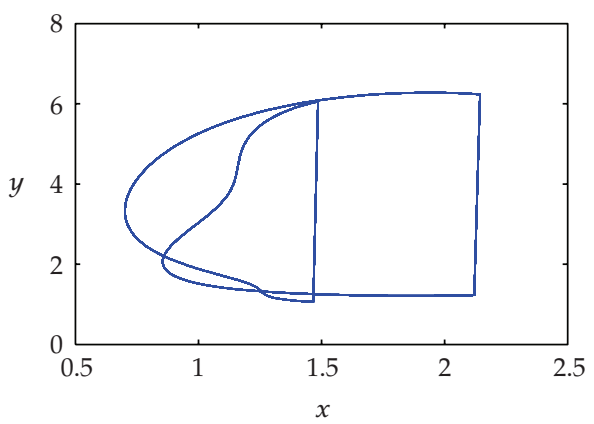

(a)

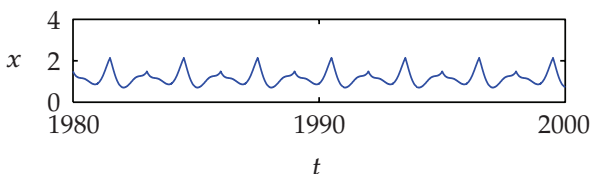

(b)

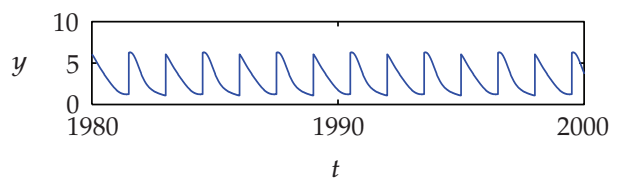

(c)

Figure 12: (a) Phase portrait of system (1.4) with $p=5, \lambda=1$, and $\tau=1.5$. (b)-(c) Time series of $x$ and $y$.

\section{Conclusion}

In this paper, we have investigated the effects of periodic forcing in the intrinsic growth rate of the prey and impulsive perturbations on a predator-prey system with the BeddingtonDeAngelis functional response. We have shown that there exists an asymptotically stable prey-free periodic solution if the magnitude $\lambda$ of seasonality is less than some critical value and have found parameter regions which system (1.4) is permanent. Numerical results have shown that system (1.4) can give birth to various kinds of dynamical behaviors. Especially, the prey and the predator can coexist even if there are seasonal effects on the prey. In addition, conditions for the stability of prey-free solution and for the permanence of Holling-type II or ration-dependent predator-prey systems have been obtained. Thus we have improved the results of [15].

\section{References}

[1] J. R. Beddington, "Mutual interference between parasites or predator and its effect on searching efficiency," Journal of Animal Ecology, vol. 44, pp. 331-340, 1975.

[2] D. L. DeAngelis, R. A. Goldstein, and R. V. O'Neill, "A model for trophic interaction," Ecology, vol. 56, pp. 881-892, 1975.

[3] G. T. Skalski and J. F. Gilliam, "Functional responses with predator interference: viable alternatives to the Holling type II mode," Ecology, vol. 82, pp. 3083-3092, 2001.

[4] M. Fan and Y. Kuang, "Dynamics of a nonautonomous predator-prey system with the BeddingtonDeAngelis functional response," Journal of Mathematical Analysis and Applications, vol. 295, no. 1, pp. 15-39, 2004. 
[5] T. W. Hwang, "Uniqueness of limit cycles of the predator-prey system with Beddington-DeAngelis functional response," Journal of Mathematical Analysis and Applications, vol. 290, pp. 113-122, 2004.

[6] J. M. Cushing, "Periodic time-dependent predator-prey systems," SIAM Journal on Applied Mathematics, vol. 32, no. 1, pp. 82-95, 1977.

[7] S. M. Moghadas and M. E. Alexander, "Dynamics of a generalized Gause-type predator-prey model with a seasonal functional response," Chaos, Solitons $\mathcal{E}$ Fractals, vol. 23, pp. 55-65, 2005.

[8] Yu. A. Kuznetsov, S. Muratori, and S. Rinaldi, "Bifurcations and chaos in a periodic predator-prey model," International Journal of Bifurcation and Chaos, vol. 2, no. 1, pp. 117-128, 1992.

[9] Z. Li, W. Wang, and H. Wang, "The dynamics of a Beddington-type system with impulsive control strategy," Chaos, Solitons \& Fractals, vol. 29, pp. 1229-1239, 2006.

[10] X. Liu and L. Chen, “Complex dynamics of Holling type II Lotka-Volterra predator-prey system with impulsive perturbations on the predator," Chaos, Solitons \& Fractals, vol. 16, pp. 311-320, 2003.

[11] B. Liu, Y. Zhang, and L. Chen, "Dynamic complexities in a Lotka-Volterra predator-prey model concerning impulsive control strategy," International Journal of Bifurcation and Chaos, vol. 15, no. 2, pp. 517-531, 2005.

[12] S. Rinaldi, S. Muratori, and Y. Kuznetsov, "Multiple attractors, catastrophes and chaos in seasonally perturbed predator-prey communities," Bulletin of Mathematical Biology, vol. 55, pp. 15-35, 1993.

[13] W. Wang, H. Wang, and Z. Li, "The dynamic complexity of a three-species Beddington-type food chain with impulsive control strategy," Chaos, Solitons \& Fractals, vol. 32, pp. 1772-1785, 2007.

[14] S. Zhang, D. Tan, and L. Chen, "Chaos in periodically forced Holling type II predator-prey system with impulsive perturbations," Chaos, Solitons E Fractals, vol. 28, no. 2, pp. 367-376, 2006.

[15] S. Zhang and L. Chen, "A study of predator-prey models with the Beddington-DeAnglis functional response and impulsive effect," Chaos, Solitons E Fractals, vol. 27, pp. 237-248, 2006.

[16] S. Gakkhar and R. K. Naji, "Chaos in seasonally perturbed ratio-dependent prey-predator system," Chaos, Solitons \& Fractals, vol. 15, pp. 107-118, 2003.

[17] G. C. W. Sabin and D. Summers, "Chaos in a periodically forced predator-prey ecosystem model," Mathematical Biosciences, vol. 113, pp. 91-113, 1993.

[18] H. Baek, "Dynamic complexities of a three-speciesbeddington-DeAngelis system with impulsive control strategy," Acta Applicandae Mathematicae.

[19] H. Baek and Y. Do, "Stability for a holling type IV food chain system with impulsive perturbations," Kyungpook Mathematical Journal, vol. 48, no. 3, pp. 515-527, 2008.

[20] H. Wang and W. Wang, "The dynamical complexity of a Ivlev-type prey-predator system with impulsive effect," Chaos, Solitons \& Fractals, vol. 38, pp. 1168-1176, 2007.

[21] S. Zhang, L. Dong, and L. Chen, "The study of predator-prey system with defensive ability of prey and impulsive perturbations on the predator," Chaos, Solitons E Fractals, vol. 23, pp. 631-643, 2005.

[22] V. Lakshmikantham, D. D. BaĬnov, and P. S. Simeonov, Theory of Impulsive Differential Equations, World Scientific, Singapore, 1989. 


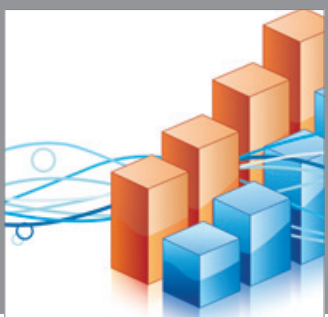

Advances in

Operations Research

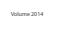

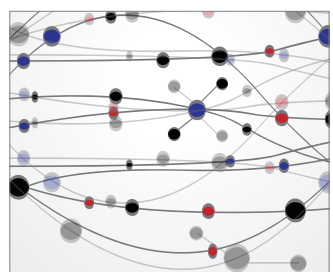

\section{The Scientific} World Journal
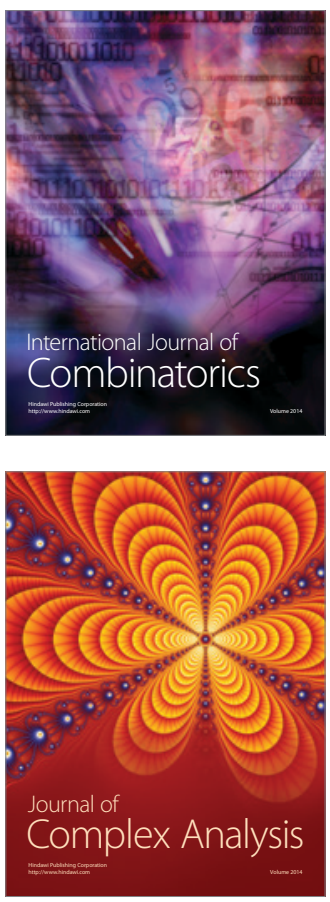

International Journal of

Mathematics and

Mathematical

Sciences
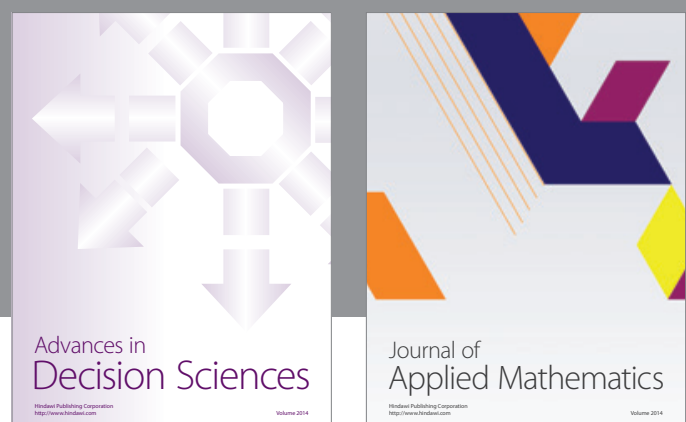

Journal of

Applied Mathematics
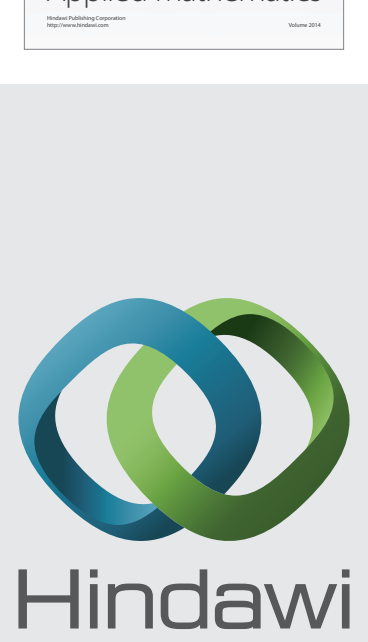

Submit your manuscripts at http://www.hindawi.com
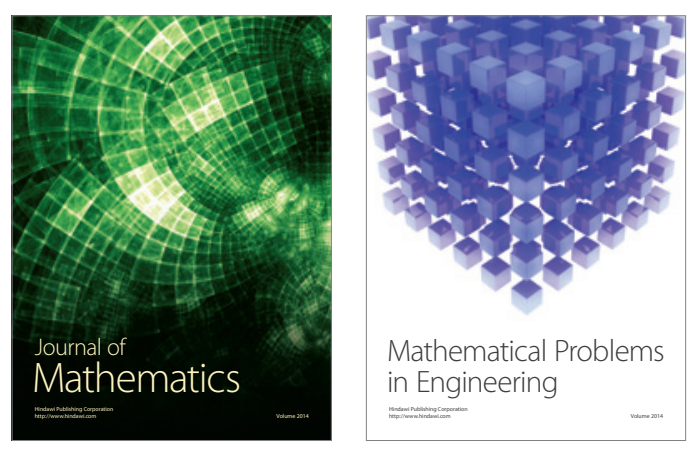

Mathematical Problems in Engineering
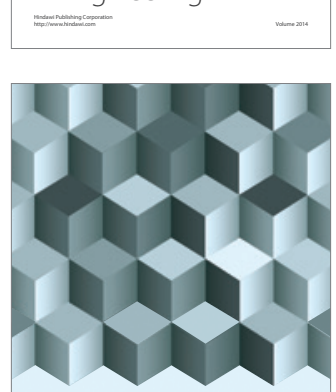

Journal of

Function Spaces
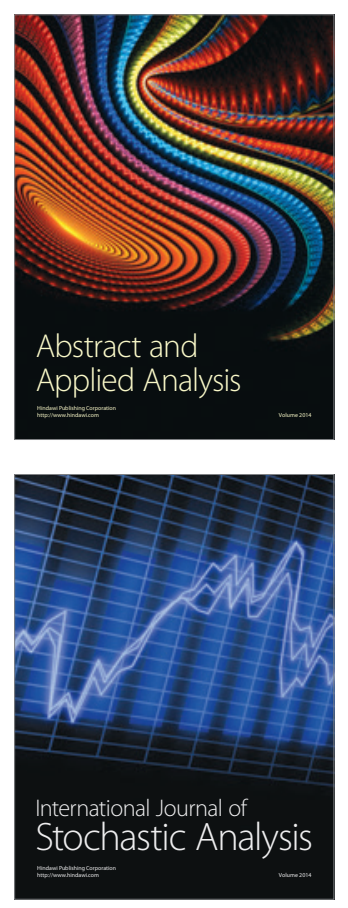

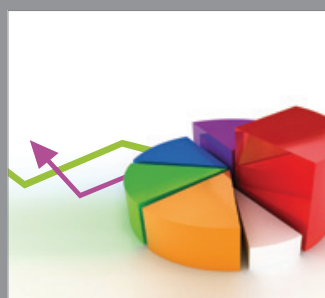

ournal of

Probability and Statistics

Promensencen
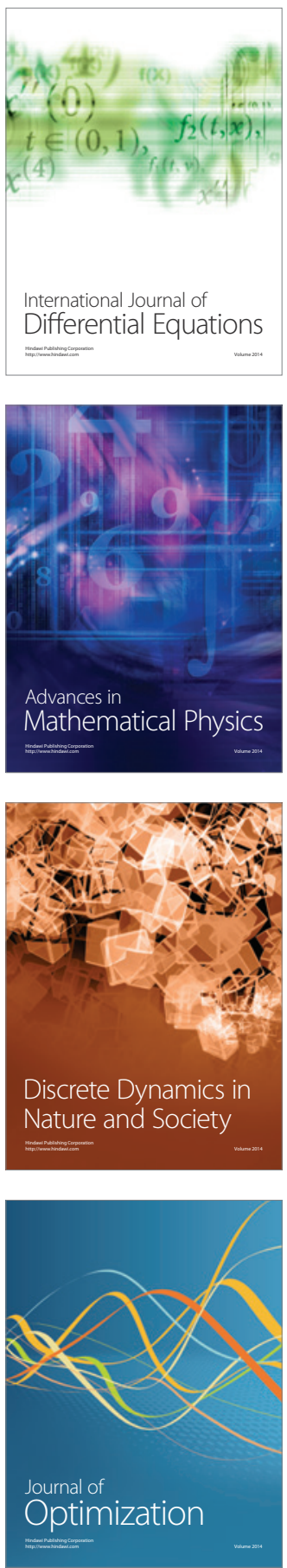